\title{
Renal background correction and measurement of split renal function: The challenge
}

\author{
Editorial Comment: EJNM-D-15-00322, M Donald Blaufox, MD, PhD
}

\author{
M. Donald Blaufox ${ }^{1}$
}

Published online: 16 November 2015

(C) Springer-Verlag Berlin Heidelberg 2015

One of the sources of error in performing quantitative nuclear medicine studies is correction for the presence of background activity surrounding the area of interest. This problem is particularly difficult to resolve in evaluating the kidney because of its location deep within the body surrounded by other highly vascular organs including the liver and spleen. The difficulty in determining quantitative individual renal function measurements accurately and subtracting the correct background activity was noted in a consensus report from the International Society of Radionuclides and Nephrourology (ISCORN) [1]. They stated that "Background subtraction is particularly critical in some methods, since intra- and extravascular activity is rapidly changing (in opposite directions) during the time when individual function is usually calculated and the contribution of each type of activity varies within different regions of interest". They also pointed out that renal depth may influence the amount of measured activity through attenuation. Taylor et al. [2] suggested improved formulas for correction of renal depth, but there remains an error from this source, which is fortunately small in most patients.

It is noted in a later consensus report from ISCORN that many of the disagreements about how and where to choose background activity to quantitate individual renal function have been resolved [3]. Unlike the previous report, the group at this time was able to achieve a consensus, but did not endorse one single method as the method of choice.

This Editorial Commentary refers to the article http://dx.doi.org/10.1007/ s00259-015-3216-1

M. Donald Blaufox

mdonald.blaufox@einstein.yu.edu

1 Department of Nuclear Medicine, Albert Einstein College of Medicine, 1695A Eastchester Road, Bronx, New York 10460, USA
Measurements of total renal function with in vivo methods remain subject to significant error [4]. The Patlak Rutland plot method was found to be accurate $[5,6]$, but it was not chosen as the preferred method. Absolute proof of the accuracy of these techniques is not available and they remain essentially theoretical approaches that have achieved some degree of general acceptance with indirect methods of validation.

The Patlak Rutland technique for correcting for renal background (interstitial and vascular) suffers from the requirement for five separate regions of interest. Regions of interest including both kidneys, two perirenal regions and the aorta are needed to calculate the correction and determine the relative renal function. Certainly a simpler technique with equivalent accuracy is desirable. The authors of the article in this issue of the European Journal of Nuclear Medicine [7] present a technique that requires only three regions of interest including the two kidneys and liver to facilitate subtraction of the background activity (including the vascular component) and calculation of individual renal function. The technique is presented with a firm theoretical basis and has been shown both to correlate very well with the Patlak Rutland technique and to be easily performed with good agreement among different observers. The data presented suggest that it may well be a useful alternative to the Patlak Rutland method, especially since by avoiding the necessity for an area of interest over the heart, the camera can be positioned lower over the patient allowing the full genitourinary system to be included in the field of view.

The paper reports the study with MAG3 and does not resolve the question as to whether it would be valid for measurement of glomerular filtration rate (GFR) with DTPA. There is also a potential source of error at very low levels of renal function due to some hepatic uptake. This does not appear to have been a problem in the present study. At the present time there is no method available to 
fully validate this technique since the absolute measurement of individual renal clearance utilizing ureteral catheterization as was practised years ago has been abandoned due to its risk and the lack of clinical justification for such precise information. Even tomographic techniques such as MRI, which allow examination of the activity only in the kidney and avoid the problem of tissue background, still have not achieved the same degree of reproducibility and accuracy as is achieved with the nuclear medicine techniques [8]. Alternatively, PET studies can be used to evaluate renal function without significant tissue background, but they are an unacceptably expensive alternative. Even after the tissue background is completely subtracted by tomography there still remains the problem of blood and interstitial fluid in the kidney, which is also part of the background and does not reflect renal function. When using GFR agents, $75-80 \%$ of the blood in the kidney at any time is merely passing through and does not reflect the GFR. An agent such as MAG3 presents less of a problem. Although the extraction is higher, only about $60-70 \%$ of the activity is being extracted by the kidney in a single pass. The extraction for these agents is even lower in the presence of impaired renal function. So even with tomographic techniques there is still the challenge of correcting for background activity within the kidney.

Given these conditions the likelihood of an absolute quantitative measurement of relative renal function with no intrinsic error remains small with the technology available to us at this time. Fortunately, it has been shown that the measurement of relative renal function using the Patlak Rutland plot for background subtraction, and even less accurate methods, still provides clinical information about the kidney and relative renal function, which can help in the care of patients. The ultimate goal is to find a methodology that is as reproducible, precise and accurate as possible within our current capabilities. Clearly this subject is a matter of general interest [9]. The article cited here offers potential improvement in relation to our current methodology by maintaining precision and accuracy at a level that is currently available and adding to it simplicity to increase the practicality of its use in clinical situations. Hopefully the data will be validated and extended to DTPA by other observers and we will find ourselves one step closer to achieving a highly accurate practical method for determining individual renal function.

\section{References}

1. Blaufox MD, Aureli M, Bubeck B, Fommei E, Piepsz A, Russell C, et al. Report of the Radionuclides in Nephrourology Committee on renal clearance. J Nucl Med. 1996;37:1883-90.

2. Taylor A, Lewis C, Giacometti A, Hall EC, Barefield KP. Improved formulas for the estimation of renal depth in adults. J Nucl Med. 1993;34:1766-9.

3. Prigent A, Cosgriff P, Gates GF, Granerus G, Fine EJ, Itoh K, et al. Consensus report on quality control of quantitative measurements of renal function obtained from the renogram: International Consensus Committee from the Scientific Committee of Radionuclides in Nephrourology. Semin Nucl Med. 1999;29:146-59.

4. Fine EJ, Michael A, Janet G, Khalid S, Donald Blaufox M. Measurement of effective renal plasma flow: a comparison of methods. J Nucl Med. 1987;28:1393-400.

5. Rutland MD. A comprehensive analysis of renal DTPA studies. I. Theory and normal values. Nucl Med Commun. 1985;6:11-20.

6. Piepsz A, Dobbeleir A, Ham HR. Effect of background correction on separate technetium-99m-DTPA renal clearance. J NucI Med. 1990;31:430-5.

7. Wesolowski MJ, Conrad GR, Šámal M, Watson G, Wanasundara SN, Babyn P, et al. A simple method for determining split renal function from dynamic 99mTc-MAG3 scintigraphic data. Eur J Nucl Med Mol Imaging. 2015. doi:10.1007/s00259-015-3216-1.

8. Durand E. Comparison of magnetic resonance imaging with radionuclide methods of evaluating the kidney. Semin Nucl Med. 2014;44:82-92.

9. De Palma D. Guest editorial. Semin Nucl Med. 2014;44:79-81. 